\title{
Substance Use Among a National Sample of Sexual and Gender Minority Adolescents: Intersections of Sex Assigned at Birth and Gender Identity
}

\author{
Ryan J. Watson, PhD, Jessica N. Fish, PhD, ${ }^{2}$ Timothy McKay, MFT, ${ }^{1}$ Samuel H. Allen, MS,2 \\ Lisa Eaton, $\mathrm{PhD},{ }^{1}$ and Rebecca M. Puhl, $\mathrm{PhD}^{1}$
}

\begin{abstract}
Purpose: We examined how substance use differed as a function of sex assigned at birth and gender identity (cisgender, transgender, or nonbinary/genderqueer) by type of substance. We sought to test whether current gender identity and sex assigned at birth were key factors in substance use among a large contemporary sample that included transgender and nonbinary/genderqueer adolescents.

Methods: We analyzed data from a large national U.S. sample of sexual and gender minority (SGM) adolescents $(n=11,129)$ collected between April and December 2017. Chi-square tests of independence were used to test whether substance use behaviors varied by sex assigned at birth and gender identity. A series of multivariate logistic regression models tested the odds of substance use by sex assigned at birth and gender identity, as well as the interaction between sex assigned at birth and gender identity.

Results: More than half of our sample reported lifetime alcohol use, and one-fourth of the sample reported lifetime marijuana use. Adolescents assigned male at birth had higher prevalence of substance use compared with adolescents assigned female at birth (AFAB). Multivariate models elucidated greater risk for most substance use outcomes for transgender adolescents compared with cisgender adolescents. We found significant interaction effects between gender identity and sex assigned at birth for recent alcohol use and lifetime and recent cigarette use among adolescents AFAB.

Conclusions: These findings have implications for stakeholders who develop nationally representative surveys, researchers who examine substance use disparities among SGM adolescents, and mental health professionals who treat underage substance use among vulnerable populations.
\end{abstract}

Keywords: alcohol use, gender identity, sexual and gender minority, substance use

\section{Introduction}

$\mathbf{E}$ LEVATED RATES OF SUBSTANCE USE remain a problem among sexual and gender minority (SGM) adolescents $^{1-3}$; higher rates of substance use affect this population disproportionately. ${ }^{4,5}$ Although a growing number of studies have examined the use of alcohol,,${ }^{6,7}$ tobacco, ${ }^{8}$ and illicit substances $^{9-11}$ among SGM populations, large-scale data that allow for within-group comparisons to elucidate potential differences across a spectrum of gender identities and sex assigned at birth have been largely unavailable. ${ }^{12}$ Consequently, we are unaware of subgroup differences in substance use across a heterogenous population of transgender and nonbinary adolescents (e.g., nonbinary adolescents assigned male at birth (AMAB), nonbinary adolescents assigned female at birth (AFAB), genderqueer adolescents, transgender boys, and transgender girls). Potential heterogeneity across gender identity, sex assigned at birth, and sexual identity among young people with respect to their substance use experiences is underexplored. ${ }^{6}$ Knowledge of substance use differences across multiple identities may alert stakeholders to subgroups at particular risk.

Gender identity is distinct from sexual identity, ${ }^{12}$ and preliminary evidence shows that health behaviors can differ appreciably across intersecting SGM identities. ${ }^{8,10}$ Only by studying the heterogeneity among SGM adolescents are we able to obtain tools for targeted prevention and intervention strategies to reduce the higher rates of compromised health

\footnotetext{
${ }^{1}$ Department of Human Development and Family Sciences, University of Connecticut, Storrs, Connecticut.

${ }^{2}$ Department of Family Science, School of Public Health, University of Maryland, College Park, Maryland.
} 
for this population. To address this, we aim to contribute to a more accurate and nuanced understanding of how health behaviors, specifically substance use, differ across diverse gender identities, including among nonbinary adolescents. That is, do differences in substance use prevalence emerge as a factor of sex assigned at birth and gender identity among SGM adolescents?

\section{Prevalence of and disparities in substance use across sexual and gender identity}

There exists a robust and growing body of literature that documents elevated risk for substance use among sexual minority adolescents. ${ }^{4,5,13}$ Studies that have traced trends in sexual orientation-related disparities in substance use have found that these disparities in alcohol, tobacco, and marijuana use have remained stable and in some cases widened over the past 15 years. ${ }^{2,14,15}$ The 2015 Youth Risk Behavior Survey (YRBS) - the first U.S. national survey to include sexual orientation-revealed stark disparities in alcohol, tobacco, and other drug use between heterosexual and sexual minority adolescents. 16

Studies that document rates of substance use among gender minority adolescents are far less common. In a large national study of 5542 13- to 18-year old adolescents, Reisner et al. ${ }^{17}$ examined differences in rates of substance use between gender minority and cisgender adolescents. The authors found elevated rates of substance use for gender minority adolescents compared to their cisgender peers (up to two times the risk of substance use). ${ }^{17}$ In the first representative study of substance use among transgender adolescents, which used the Biennial Statewide California Healthy Kids Survey (CHKS; 2013-2015), Day et al. ${ }^{1}$ found that transgender adolescents reported higher prevalence of lifetime and past 30-day substance use (alcohol use, heavy episodic drinking, cigarette use, marijuana use, use of other drugs, and polysubstance use) than their cisgender peers. Comparatively, 30-day heavy episodic drinking was 3times higher and marijuana use was 2.5 times higher for transgender adolescents compared to cisgender adolescents. ${ }^{1}$

\section{Improving specificity in documenting SGM substance use}

SGM-related disparities are oftentimes explained by the minority stress model, ${ }^{18,19}$ although empirical support for the application of the model to transgender-specific health risk is still emerging. ${ }^{17}$ Despite a growing body of SGM substance use literature, studies are slow to systematically explore patterns of substance use across distinct identities. ${ }^{20}$ Given the need for population-specific prevention and intervention efforts, we aimed to address this gap in the field by identifying unique patterns of substance use across subcategories of SGM adolescents. We estimated the prevalence of alcohol, tobacco, and marijuana use behaviors across groups defined by diverse gender identities and sex assigned at birth.

\section{Methods}

\section{Study design, participant recruitment, and data} cleaning procedures

Data are from the LGBTQ National Teen Survey, which was designed to better understand the day-to-day experi- ences of SGM racially diverse adolescents, collected between April and December 2017. Adolescents were eligible if they reported being ages 13-17 years, had a sexual and/or gender minority identity, resided in the United States, could complete an online survey, and could read English. The survey was hosted on Qualtrics.com (Provo, Utah and Seattle, Washington) and was used to collect data from anonymous participants recruited through social media with marketing assistance from social influencers. The Human Rights Campaign (HRC) posted Facebook and Twitter messages with a link to the survey. Informed assent was achieved through the Study Information sheet presented before the Qualtrics survey; all study protocols, including a waiver of parental consent, were approved by the University of Connecticut Institutional Review Board. More information regarding the study design can be found elsewhere. ${ }^{21,22}$

In total, 20,306 eligible participants started the survey. Among those, 3006 (14.8\%) participants did not complete the initial demographic information and were excluded from all data analyses. Open-ended responses were examined; we deleted suspicious entries not previously captured by the mischievous responders screening process (e.g., referring to oneself as Donald Trump; $n=79$ ). Although the survey was designed to prevent bots from completing the survey, a post hoc mischievous responder's sensitivity analysis $^{23}$ was also conducted on the data from eligible responders $(n=74$ removed). Duplicate surveys $(n=12)$ and those where a participant began a survey but terminated it before completion and then completed a new survey $(n=23)$ were deleted by manually identifying identical survey responses. Completion time for participants who reached the end of the survey was on average 43.3 minutes (median $=28.2$ minutes).

Participants were eligible for HRC wristbands and entry into an Amazon.com gift card drawing for completing the survey. Of the larger study, a final total of 17,112 participants was represented by $4740(27.7 \%)$ adolescents AMAB, $12,372(72.3 \%)$ adolescents AFAB, a majority of White adolescents $(n=10,225 ; 59.8 \%), 4079(23.8 \%)$ cisgender boys, $7396(43.2 \%)$ cisgender girls, $1404(8.2 \%)$ transgender boys, $185(1.1 \%)$ transgender girls, $3573(20.9 \%)$ nonbinary adolescents $\mathrm{AFAB}$, and 475 (2.8\%) nonbinary adolescents AMAB. The sample for this article included adolescents who provided valid data on gender identity measures and who were not missing on all substance use outcomes ( $n=11,129$ adolescents).

\section{Measures}

Sex assigned at birth. Participants were asked, "What sex were you assigned at birth?" Response options were male and female.

Gender identity. Participants were asked, "What is your current gender identity?" Response options were male, female, trans male/boy, trans female/girl, nonbinary, genderqueer/ gender nonconforming, and different identity, with a writein response. From these options we created three mutually exclusive groups of adolescents-cisgender, transgender, and nonbinary/genderqueer. Adolescents with concordant sex assigned at birth and gender identities were coded as cisgender. Adolescents who reported a gender identity that was different from their sex assigned at birth and exclusively 
chose the "trans male/boy" or "trans female/girl" options were coded as transgender-we conceptualize these adolescents as "binary" transgender youth. A third group of adolescents was coded as "nonbinary/genderqueer" given that they chose a gender identity that was different from their sex assigned at birth and selected "nonbinary," "genderqueer/ gender nonconforming," or a combination of gender identities that included a nonbinary response (e.g., trans male/boy and nonbinary). Adolescents who reported a write-in response were back-coded when appropriate and, if not, analyzed to determine if their identity was to be characterized as binary or nonbinary.

Substance use. We measured alcohol, marijuana, and cigarette smoking in a way that mirrored questions asked in the 2015 YRBS. ${ }^{16}$ All substance use items were recoded to reflect no use $=0$ and any use $=1$.

Lifetime marijuana use. One question asked, "During your life, on how many days have you had marijuana?" Response options ranged from 0 (0 days) to 6 (100 or more days).

Lifetime alcohol use. One question asked, "During your life, on how many days have you had at least one drink of alcohol?" Response options ranged from 0 (0 days) to 6 (100 or more days).

Lifetime cigarette use. We asked, "Have you ever tried cigarette smoking, even one or two puffs?" Response options were 0 (no) and 1 (yes).

Recent marijuana use. One item asked, "During the past 30 days, how many times did you use marijuana?" Response options ranged from 0 ( 0 times) to 4 (40 or more times).

Recent alcohol use. One item asked, "During the past 30 days, on how many days did you have at least one drink of alcohol?" Response options ranged from 0 (0 days) to 6 (all 30 days).

Recent cigarette use. One item asked, "During the past 30 days, on how many days did you smoke cigarettes?" Response options ranged from 0 (0 days) to 6 (all 30 days).

Recent binge drinking. One item asked, "During the past 30 days, on how many days did you have five or more drinks of alcohol in a row, that is, within a couple of hours?" Response options ranged from 0 (0 days) to 6 (all 30 days).

\section{Covariates}

Sexual identity. One item asked, "How do you describe your sexual identity?" Participants could choose one of the following options, "Gay or Lesbian," "Bisexual," "Straight, that is, not gay," or "Something else." In this study, "straight" adolescents were included in the "Something else" group.

Race/ethnicity. One check-all-that-applies item asked participants, "How would you describe yourself?" Response options were "White, non-Hispanic," "Non-Latino Black or African American," "American Indian or Alaska Native," "Asian or Pacific Islander," "Latino, Hispanic, or Mexican
American," and "Other." When participants checked more than one box, they were categorized as having "Multiple Identities."

Parental education. Parental education was measured by the highest value from one of two items that stated, "Please indicate the highest level of education of your first [second] parent/primary caregiver." Response options were, "Less than high school or GED," "High school or GED," "Vocational/Technical School (2 years)," "Some college," "College graduate," and "Postgraduate degree or higher." These values were recoded to less than high school, high school, some college (including some college and vocational/technical school), and college graduate or more (including college graduate and postgraduate degree or higher).

\section{Age. Participants' age was measured in years.}

Region. Participants' region of residence was measured by coding their response to "What state do you live in?" to one of four options: "Northeast," "Midwest," "South," and "West."

\section{Plan of analysis}

All analyses were conducted in Stata 15 (2017; StataCorp LLC, College Station, TX). We first estimated the prevalence of each specific substance use behavior in the full sample. Next, using chi-square tests of independence, we tested whether the prevalence of substance use behaviors (e.g., alcohol, marijuana, and cigarette) varied by sex assigned at birth and gender identity. We then conducted a series of multivariate logistic regression models to test the odds of substance use by sex assigned at birth and gender identity, as well as the interaction between sex assigned at birth and gender identity. Given significant interactions, we also estimated sex assigned at birth stratified models to assess gender identity differences in substance use behaviors. Following the testing of interactions, we calculated predicted probabilities of substance use for subgroups from significant interactions. All models were adjusted for sexual identity, race/ethnicity, parental education, age, and region. Statistical tests at $p<0.05$ were deemed statistically significant for all analyses. We used multiple imputations with 50 replications to account for missing data on covariates.

\section{Results}

Demographic characteristics of the participants are presented in Table 1. Respondents represented diverse subgroups of SGM adolescents from all 50 states across the United States. Among adolescents AMAB, 85.71\% identified as cisgender, $4.61 \%$ as transgender, and $9.69 \%$ as nonbinary/genderqueer. Among adolescents AFAB, 58.57\% identified as cisgender, $11.46 \%$ as transgender, and $29.97 \%$ as nonbinary/genderqueer. The majority of the sample was White $(65 \%)$; the most common racial minority groups were multiple identities (13\%) and Latinx $(10 \%)$. The sample was geographically diverse, with $18 \%$ living in the Northeast, $23 \%$ in the Midwest, 36\% in the South, and $21 \%$ in the West. Approximately two-thirds of respondents' parents had at least a college degree (66.9\%). The largest 
Table 1. Demographic Characteristics of the Sample

\begin{tabular}{|c|c|c|c|c|c|c|}
\hline & \multicolumn{2}{|c|}{ Total Sample } & \multicolumn{2}{|c|}{$A M A B$} & \multicolumn{2}{|c|}{$A F A B$} \\
\hline & $\mathrm{n}$ & $\%$ & $\mathrm{n}$ & $\%$ & $\mathrm{n}$ & $\%$ \\
\hline \multicolumn{7}{|l|}{ Sex assigned at birth } \\
\hline Male & 2736 & 24.58 & & & & \\
\hline Female & 8393 & 75.42 & & & & \\
\hline \multicolumn{7}{|l|}{ Gender identity } \\
\hline Cisgender & 7261 & 65.24 & 2345 & 85.71 & 4916 & 58.57 \\
\hline Transgender & 1088 & 9.78 & 126 & 4.61 & 962 & 11.46 \\
\hline Nonbinary/genderqueer & 2780 & 24.98 & 265 & 9.69 & 2515 & 29.97 \\
\hline \multicolumn{7}{|l|}{ Sexual identity } \\
\hline Gay/lesbian & 4084 & 37.30 & 1821 & 67.25 & 2263 & 27.46 \\
\hline Bisexual & 3741 & 34.17 & 654 & 24.15 & 3087 & 37.46 \\
\hline Something else & 3124 & 28.53 & 233 & 8.60 & 2891 & 35.08 \\
\hline \multicolumn{7}{|l|}{ Race/ethnicity } \\
\hline White & 7281 & 65.49 & 1712 & 62.60 & 5569 & 66.44 \\
\hline Black & 505 & 4.54 & 148 & 5.41 & 357 & 4.26 \\
\hline Latinx & 1147 & 10.32 & 344 & 12.58 & 803 & 9.58 \\
\hline Asian & 429 & 3.86 & 121 & 4.42 & 308 & 3.67 \\
\hline Multiple identities & 1516 & 13.64 & 349 & 12.76 & 1167 & 13.92 \\
\hline Other & 239 & 2.15 & 61 & 2.23 & 178 & 2.12 \\
\hline \multicolumn{7}{|l|}{ Parental education } \\
\hline Less than high school & 321 & 3.08 & 116 & 4.50 & 205 & 2.61 \\
\hline High school & 1325 & 12.71 & 369 & 14.3 & 956 & 12.19 \\
\hline Some college & 1801 & 17.28 & 436 & 16.9 & 1365 & 17.40 \\
\hline College degree or more & 6976 & 66.93 & 1659 & 64.3 & 5317 & 67.79 \\
\hline \multicolumn{7}{|l|}{ Age } \\
\hline 13 & 829 & 7.45 & 92 & 3.36 & 737 & 8.78 \\
\hline 14 & 1652 & 14.84 & 289 & 10.56 & 1363 & 16.24 \\
\hline 15 & 2323 & 20.87 & 529 & 19.33 & 1794 & 21.37 \\
\hline 16 & 2913 & 26.17 & 793 & 28.98 & 2120 & 25.26 \\
\hline 17 & 3412 & 30.66 & 1033 & 37.76 & 2379 & 28.35 \\
\hline \multicolumn{7}{|l|}{ Region } \\
\hline Northeast & 2028 & 18.22 & 512 & 18.71 & 1516 & 18.06 \\
\hline Midwest & 2584 & 23.22 & 623 & 22.77 & 1961 & 23.36 \\
\hline South & 4080 & 36.66 & 987 & 36.07 & 3093 & 36.85 \\
\hline West & 2437 & 21.90 & 614 & 22.44 & 1823 & 21.72 \\
\hline
\end{tabular}

$\mathrm{AFAB}$, assigned female at birth; $\mathrm{AMAB}$, assigned male at birth.

proportion of respondents was identified as gay/lesbian (37\%) followed by bisexual (34\%) and something else (28\%).

\section{Prevalence of substance use by sex assigned at birth and gender identity}

Table 2 presents the prevalence of substance use by sex assigned at birth and gender identity. Among the full sample, $\sim 55 \%$ of SGM adolescents reported lifetime alcohol use, $27 \%$ reported recent alcohol use, and nearly $10 \%$ had recently engaged in binge drinking. Nearly $27 \%$ of adolescents indicated lifetime marijuana use, $14 \%$ reported recent marijuana use, $22 \%$ of adolescents reported lifetime cigarette use, and $7 \%$ reported recent cigarette use.

We found significant differences in recent alcohol use, recent binge drinking, lifetime and recent marijuana use, and lifetime cigarette use by sex assigned at birth (Table 2). Adolescents $\mathrm{AMAB}$ indicated greater prevalence of substance use relative to adolescents AFAB. Gender identity was significantly related to all substance use behaviors. Generally, transgender adolescents evidenced higher prevalence rates of lifetime alcohol, as well as lifetime and recent marijuana and cigarette use, compared to cisgender and nonbinary/genderqueer adolescents, who did not differ from one another. Conversely, nonbinary/genderqueer adolescents reported the lowest prevalence of recent alcohol use and binge drinking relative to cisgender and transgender adolescents.

Substance use behavior differences across groups simultaneously defined by sex assigned at birth and gender identity are presented in Table 3. Generally, transgender adolescents $\mathrm{AFAB}$ and cisgender adolescents $\mathrm{AMAB}$ reported the highest prevalence rates of lifetime and recent alcohol use, although transgender and cisgender adolescents AMAB reported the highest prevalence of recent binge drinking. Transgender adolescents AMAB and AFAB reported the highest rates of lifetime and recent marijuana use relative to other groups, and transgender adolescents AFAB reported the highest rates of cigarette use relative to all other groups defined by sex assigned at birth and gender identity. Nonbinary/ genderqueer adolescents AFAB typically reported lower 


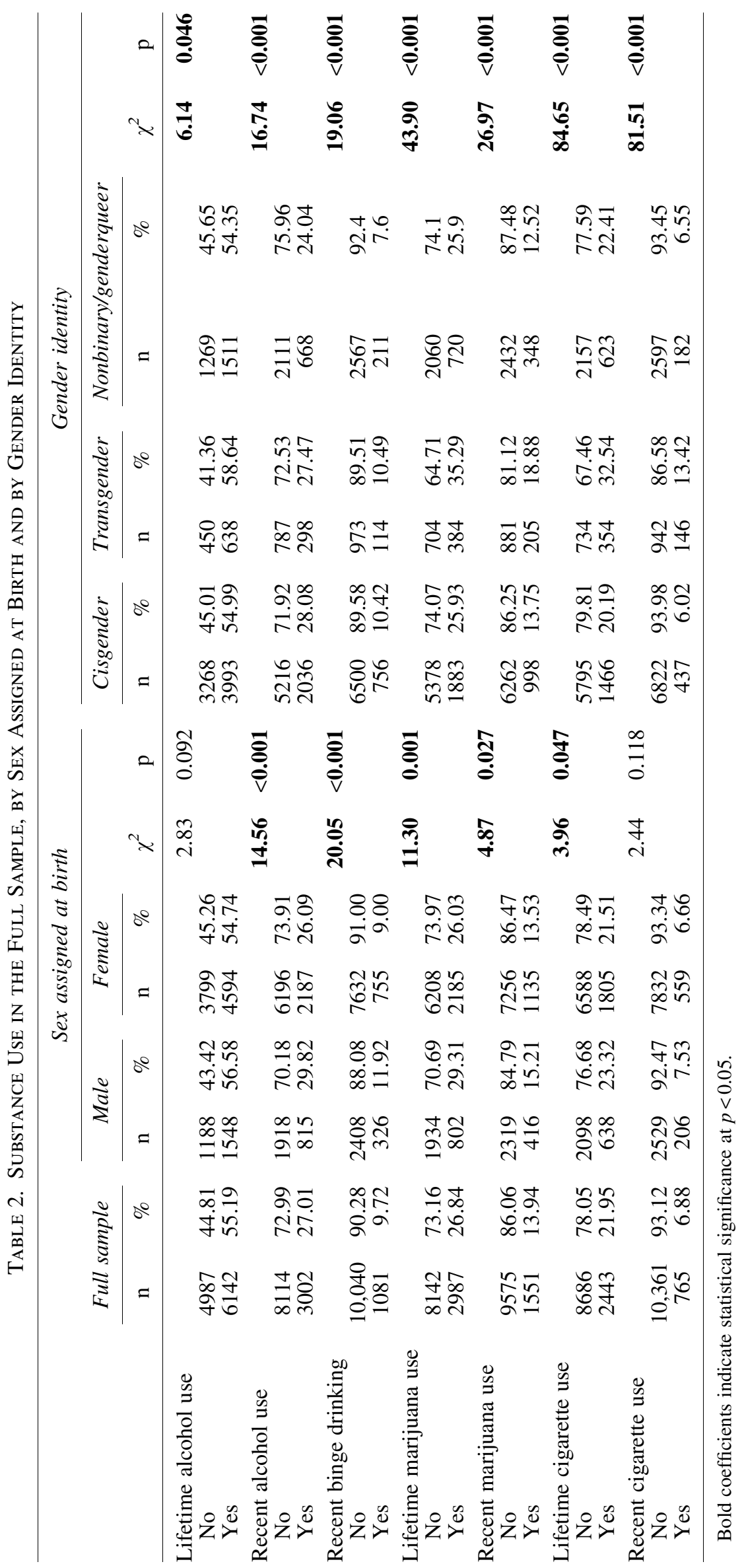




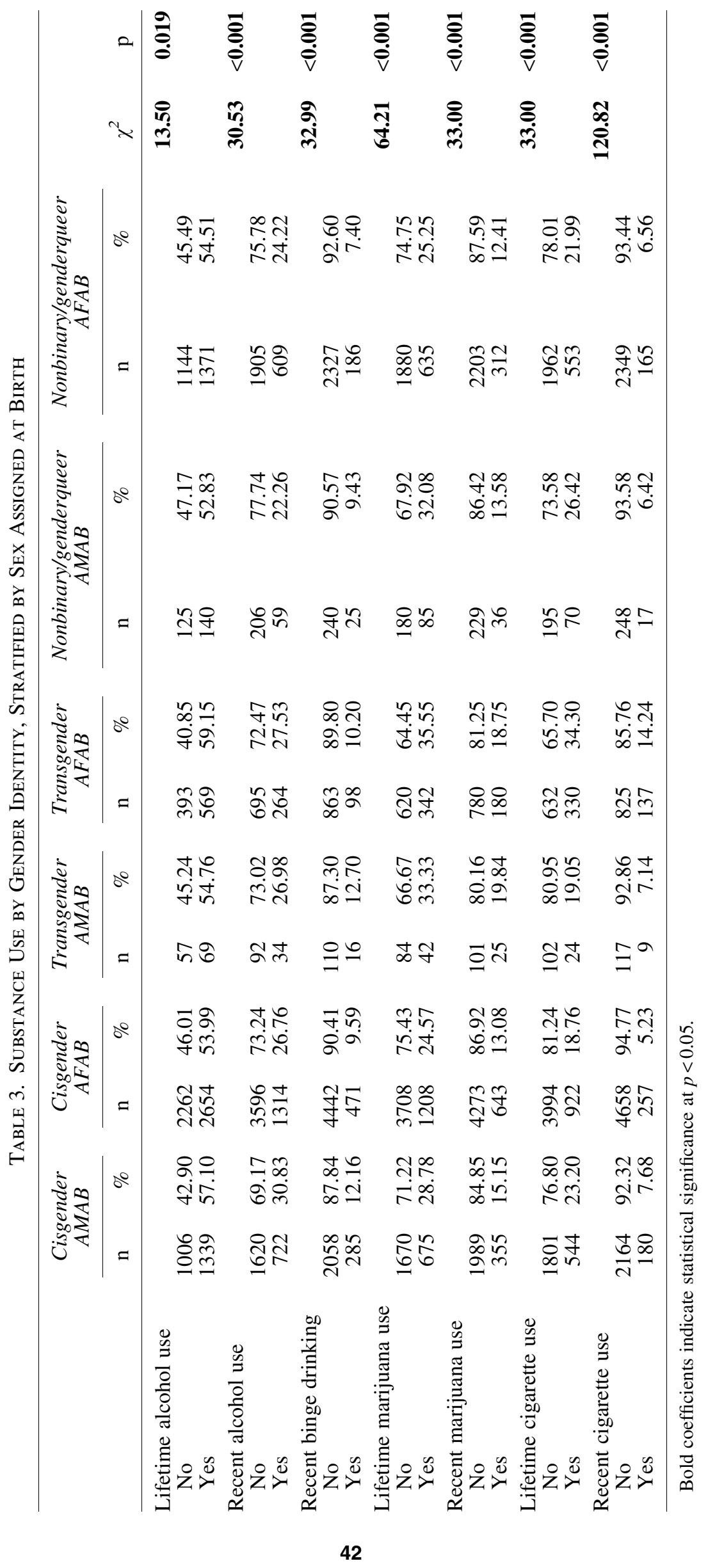




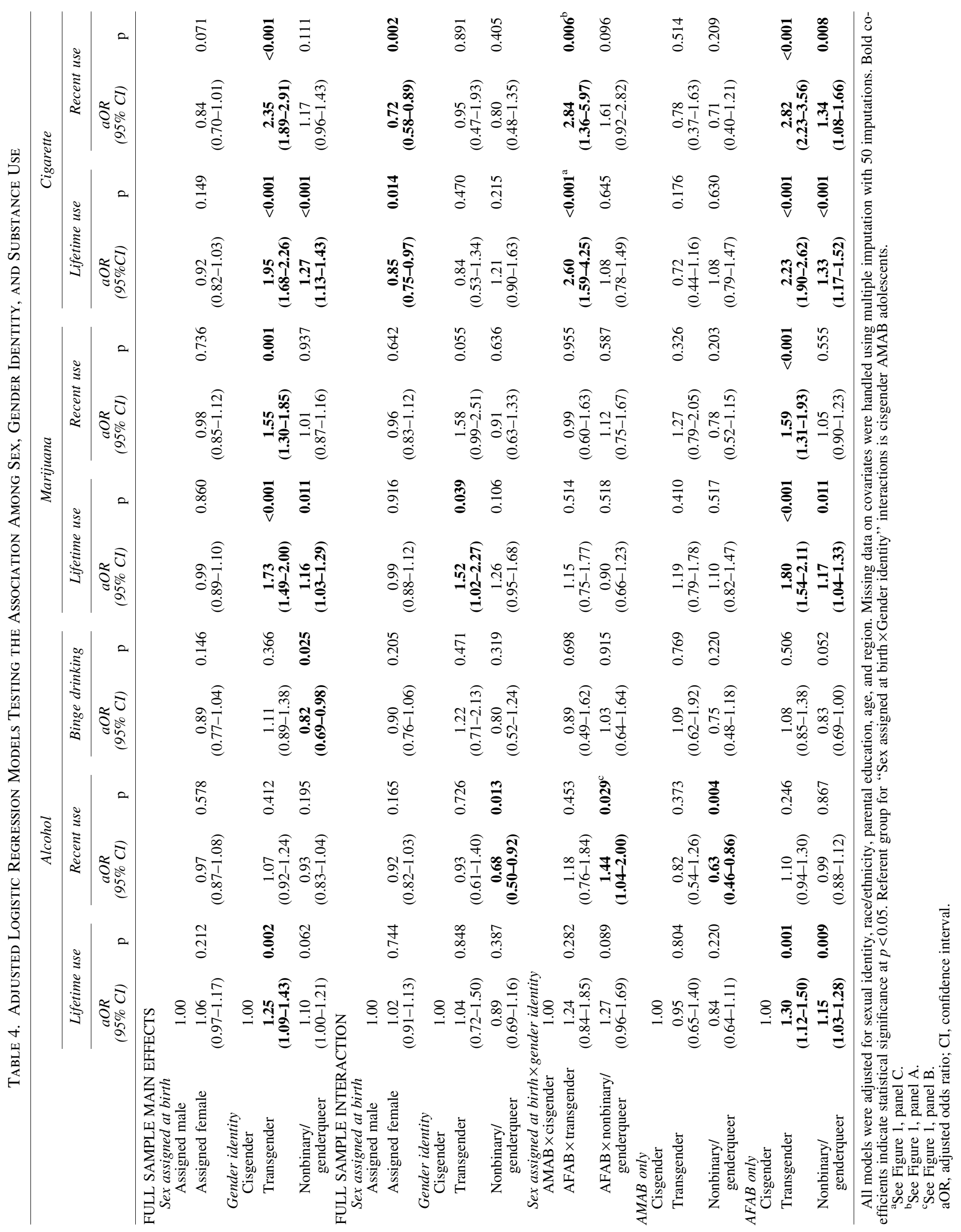


prevalence rates of all substances relative to transgender adolescents and similar or lower prevalence rates of all substances relative to cisgender adolescents of the same sex assigned at birth.

Table 4 displays results from adjusted logistic regression models, across all substance use outcomes, testing (1) the independent effects of sex assigned at birth and gender identity, (2) interaction effects of sex assigned at birth and gender identity, and (3) the independent effect of gender identity in models that are stratified by sex assigned at birth. Among the full sample, gender identity was significantly associated with increased odds of substance use. Specifically, compared to cisgender sexual minority adolescents, transgender adolescents had greater odds of lifetime alcohol use, as well as lifetime and recent marijuana and cigarette use. Nonbinary/genderqueer adolescents had lower odds of recent binge drinking, but greater odds of lifetime marijuana and cigarette use than cisgender sexual minority adolescents.

Interaction effects between sex assigned at birth and gender identity were significant for recent alcohol use, as well as lifetime and recent cigarette use. Nonbinary/genderqueer adolescents AFAB were more likely to report recent alcohol use than their cisgender AMAB counterparts. Transgender adolescents AFAB were more than twice as likely to report both lifetime and recent cigarette use than their cisgender AMAB peers (Table 4).

Given significant interaction effects between sex assigned at birth and gender identity, we also tested gender identity differences in the odds of substance use in models stratified by sex assigned at birth. No statistically significant gender identity differences were found in the odds of substance
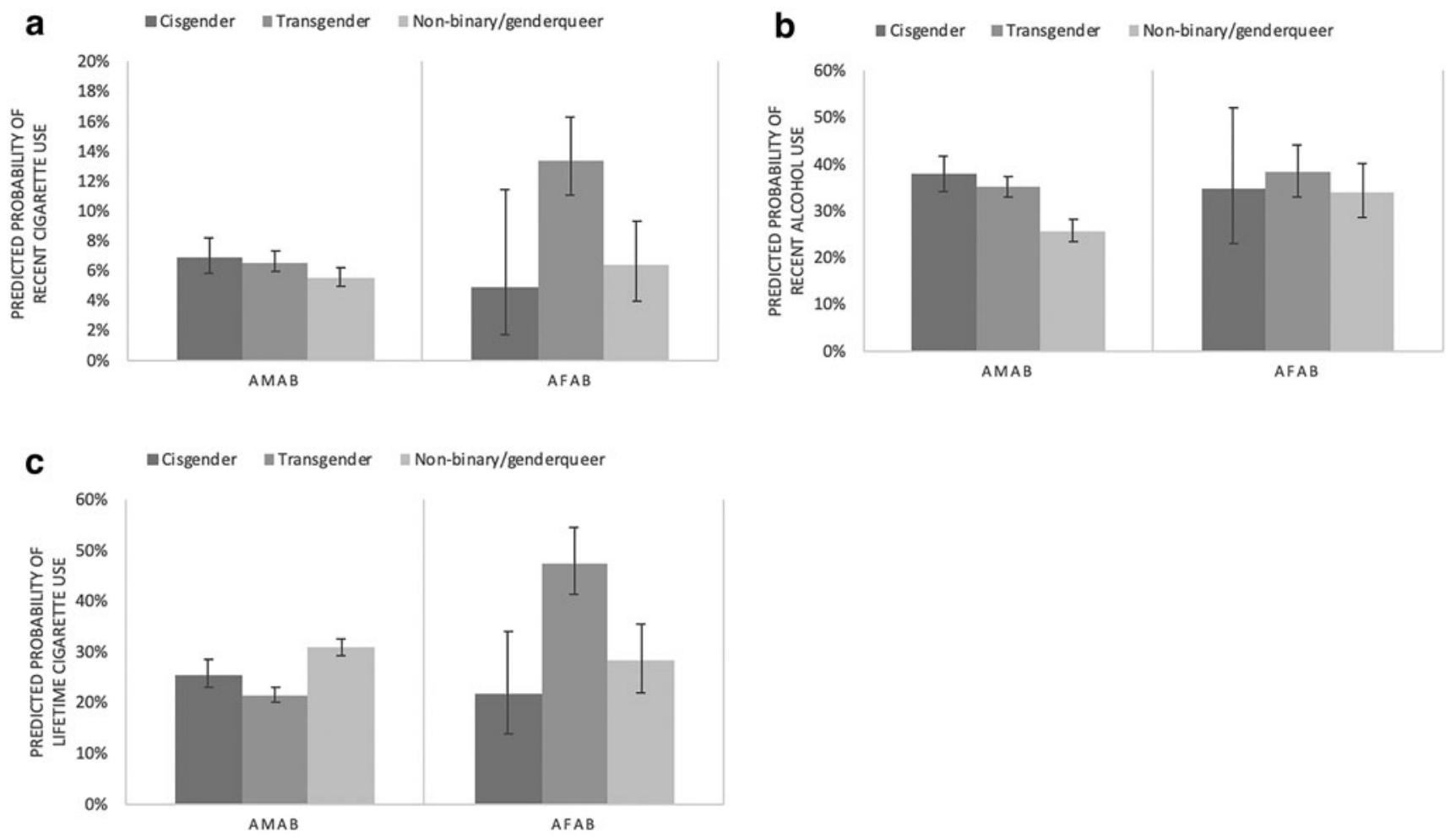

use behaviors between adolescents AMAB with the exception of recent alcohol use, where nonbinary/genderqueer adolescents AMAB had lower odds of recent alcohol use than their cisgender AMAB peers. Conversely, among adolescents AFAB, both transgender and nonbinary/genderqueer respondents had greater odds of lifetime alcohol use, lifetime marijuana use, and lifetime and recent cigarette use compared to their cisgender AFAB counterparts. In addition, transgender adolescents AFAB had greater odds of recent marijuana use than cisgender adolescents AFAB. Finally, Figure 1 illustrates the predicted probabilities of recent cigarette use, recent alcohol use, and lifetime cigarette use based on odds ratio interactions (i.e., Sex assigned at birth $\times$ Gender identity) from Table 4.

\section{Discussion}

We assessed the prevalence of lifetime and recent alcohol, marijuana, and cigarette use by sex assigned at birth and gender identity using a large national sample of SGM adolescents. Our findings suggest that there are distinct differences in the prevalence of substance use on the basis of sex assigned at birth and gender identity and at the intersection of sex assigned at birth and gender identity. It is important to note that cisgender AMAB and AFAB sexual minority adolescents constituted our referent group for $\mathrm{AMAB}$ and $\mathrm{AFAB}$ models, respectively, and that cisgender sexual minority adolescents are already at elevated risk for substance use above and beyond cisgender heterosexual adolescents. In addition to this established risk, we also found that the pattern of risk on the basis of sex assigned at birth

FIG. 1. Predicted probabilities of substance use outcomes by sex assigned at birth and gender identity, adjusted for sexual identity, race/ethnicity, parental education, age, and region. (a) Predicted probabilities of recent cigarette use, (b) predicted probabilities of recent alcohol use, and (c) predicted probabilities of lifetime cigarette use. 
and gender identity among gender minority adolescents varied depending on the type of substance. Notably, we observed elevated risk for cigarette smoking (lifetime and recent) for transgender adolescents AFAB (collapsed across sexual identities). These different patterns underscore the need for scholars to continue examining health behaviors at the intersection of sex assigned at birth and gender identity and the mechanisms that drive these differences.

Partly to assess the utility and validity of these data, we compared our findings to other national datasets and found both noteworthy similarities and differences. Two datasets allowed us to contextualize the findings from this study, including statewide data from the 2013-2015 CHKS $^{1}$ and the statewide 2016 Minnesota Student Survey (MSS). ${ }^{24}$ Overall, we found that transgender adolescents in our study reported a lower prevalence of lifetime substance use than that reported in representative population-based samples of adolescents. Using data from the CHKS, Day et al. ${ }^{1}$ found that $56 \%$ of transgender adolescents reported lifetime alcohol use, $35 \%$ reported cigarette use, and $40 \%$ reported marijuana use; among transgender adolescents in our sample, prevalence rates were $59 \%, 33 \%$, and 35\%, respectively. In addition, Eisenberg et al. ${ }^{24}$ used the MSS (9th and 11th graders) and found that $16 \%$ of transgender and gender nonconforming girls and $9 \%$ of boys reported past-30-day binge drinking; we found that $13 \%$ of transgender girls and $10 \%$ of transgender boys reported recent binge drinking. Because the CHKS only asked about transgender identity and the MSS study combined adolescents with transgender or gender nonconforming identities, these comparisons only include our findings for transgender adolescents (i.e., exclude nonbinary/genderqueer adolescents).

Our study is unique in that we disaggregated (binary) transgender adolescents from nonbinary/genderqueer adolescents, the latter of whom were at lower risk for binge drinking, but higher risk for lifetime marijuana and cigarette use compared to their cisgender counterparts (Table 4, full sample main effects). Taken together, we found that transgender adolescents in our study were at lower risk for using substances compared to transgender/gender-nonconforming adolescents in other representative samples. ${ }^{1,24}$ This is perhaps because the adolescents who responded to our online survey may have been better connected to social networks, such as those targeted as part of our recruitment. Online samples might reach adolescents who are less at risk, as this sampling technique typically misses adolescents who are homeless or do not have consistent access to the Internet.

These findings have implications for stakeholders interested in reducing disparities and improving health among SGM individuals. First, our study implies that a dual understanding of sex in combination with gender identity is important for the proper identification of substance use risk and potential intervention among SGM adolescents. Therefore, stakeholders (e.g., physicians, practitioners, and researchers) need to be aware of and properly assess for both when engaging SGM adolescents in research and practice. Second, relative to cisgender sexual minority adolescents, we found several instances where transgender and nonbinary/ genderqueer adolescents were at elevated risk for substance use. Physicians and intervention scholars should recognize that transgender adolescents may require specific substance use-related prevention strategies. Finally, our study confirms the importance for researchers to include comprehensive and diverse measures of sexual orientation and gender identities in their studies. Given that our findings underscore significant differences among SGM subgroups, scholars who study substance use should consider measuring the diversity of SGM identities in their epidemiological, prevention, and intervention scholarship.

\section{Limitations}

Although the present study uses a large dataset and provides a unique perspective on the heterogeneity of substance use behaviors among SGM adolescents, the data are not nationally representative. In addition, the data are crosssectional, which limits our ability to explore substance use across time and within the context of the gender transition process or general age-related changes in substance use. The online data collection process likely prevented SGM adolescents who were experiencing homelessness or living in shelters without Internet access to participate. We were also underpowered to detect differences in substance use behaviors between cisgender and gender minority adolescents who were AMAB. With greater power, we might expect to see interaction effects between sex assigned at birth and gender identity that enumerate differences among adolescents AMAB - hopefully future work can address this. Although necessary to retain power, the decision to dichotomize substance use measures eliminated our ability to test differences in the frequency of alcohol, marijuana, and cigarette use among SGM adolescents. Future studies should consider models that assess frequencies as a unique indicator risk above and beyond general reports of use. Finally, we did not include other illicit substances as outcomes in this articleyet there are serious concerns about opioid and other "hard drug" use among vulnerable populations. This deserves focused attention moving forward.

\section{Conclusion}

Our findings offer novel insights through a comprehensive and systematic assessment of patterns of substance use among gender minority adolescents using a large sample of SGM adolescents; they also provide a first step in understanding within-group differences in SGM substance use and, in particular, differential risk for distinct substance use outcomes across groups defined by sex assigned at birth and gender identity. Our findings can inform future studies by serving as a framework for the assessment and recognition of the multiplicity of sexual identity, gender identity, and sex assigned at birth. Population-based surveys should continue their commitment to measuring sexual orientation and gender identity and the diversity therein.

\section{Acknowledgments}

The authors acknowledge the important contributions of Ellen Kahn, Gabe Murchison, and Liam Miranda in their support, conceptualization, and management related to the LGBTQ National Teen Study.

\section{Disclaimer}

This research uses data from the LGBTQ National Teen Study, designed by R.J.W. and R.M.P. in collaboration 
with the HRC, and supported by the Office of the Vice President for Research at the University of Connecticut. Any interpretations and opinions expressed herein are solely those of the authors and may not reflect those of the NIH or the CDC.

\section{Author Disclosure Statement}

No competing financial interests exist.

\section{Funding Information}

Research reported in this publication was supported by the National Institute on Drug Abuse of the National Institutes of Health under award no. R03DA046827, SGM Youth Substance Use: Subgroup Differences, Risk, and Protective Factors. This research was funded, in part, by the Office of the Vice President for Research at the University of Connecticut and in partnership with the HRC. J.N.F. acknowledges support from the University of Maryland Prevention Research Center cooperative agreement no. U48DP006382 from the Centers for Disease Control and Prevention (CDC) and the Eunice Kennedy Shriver National Institute of Child Health and Human Development grant P2CHD041041, Maryland Population Research Center. R.J.W. acknowledges support from the National Institute on Drug Abuse grant K01DA047918.

\section{References}

1. Day JK, Fish JN, Perez-Brumer A, et al.: Transgender youth substance use disparities: Results from a population-based sample. J Adolesc Health 2017;61:729F-735F.

2. Fish JN, Baams L: Trends in alcohol-related disparities between heterosexual and sexual minority youth from 2007 to 2015: Findings from the Youth Risk Behavior Survey. LGBT Health 2018;5:359-367.

3. Fish JN, Turner B, Phillips G 2nd, Russell ST: Cigarette smoking disparities between sexual minority and heterosexual youth. Pediatrics 2019;143:e20181671.

4. Marshal MP, Friedman MS, Stall R, et al.: Sexual orientation and adolescent substance use: A meta-analysis and methodological review. Addiction 2008;103:546-556.

5. Plöderl M, Tremblay P: Mental health of sexual minorities. A systematic review. Int Rev Psychiatry 2015;27:367-385.

6. Talley AE, Gilbert PA, Mitchell J, et al.: Addressing gaps on risk and resilience factors for alcohol use outcomes in sexual and gender minority populations. Drug Alcohol Rev 2016;35:484-493.

7. Fish JN, Schulenberg JE, Russell ST: Sexual minority youth report high-intensity binge drinking: The critical role of school victimization. J Adolesc Health 2019;64:186-193.

8. Wheldon CW, Watson RJ, Fish JN, Gamarel K: Cigarette smoking among youth at the intersection of sexual orientation and gender identity. LGBT Health 2019;6:235-241.

9. Hatzenbuehler ML, Jun HJ, Corliss HL, Bryn Austin S: Structural stigma and sexual orientation disparities in adolescent drug use. Addict Behav 2015;46:14-18.

10. Demant D, Oviedo-Trespalacios O, Carroll JA, et al.: Do people with intersecting identities report more high-risk alcohol use and lifetime substance use? Int J Public Health 2018;63:621-630.
11. Newcomb ME, Birkett M, Corliss HL, Mustanski B: Sexual orientation, gender, and racial differences in illicit drug use in a sample of US high school students. Am J Public Health 2014;104:304-310.

12. Institute of Medicine (US) Committee on Lesbian, Gay, Bisexual, and Transgender Health Issues and Research Gaps and Opportunities. The Health of Lesbian, Gay, Bisexual, and Transgender People: Building a Foundation for Better Understanding. Washington, DC: The National Academies Press, 2011.

13. Goldbach JT, Tanner-Smith EE, Bagwell M, Dunlap S: Minority stress and substance use in sexual minority adolescents: A meta-analysis. Prev Sci 2014;15:350-363.

14. Fish JN, Watson RJ, Porta CM, et al.: Are alcohol-related disparities between sexual minority and heterosexual youth decreasing? Addiction 2017;112:1931-1941.

15. Watson RJ, Goodenow C, Porta C, et al.: Substance use among sexual minorities: Has it actually gotten better? Subst Use Misuse 2018;53:1221-1228.

16. Kann L, Olsen EO, McManus T, et al.: Sexual identity, sex of sexual contacts, and health-related behaviors among students in grades 9-12-United States and selected sites, 2015. MMWR Surveill Summ 2016;65:1-202.

17. Reisner SL, Greytak EA, Parsons JT, Ybarra ML: Gender minority social stress in adolescence: Disparities in adolescent bullying and substance use by gender identity. J Sex Res 2015;52:243-256.

18. Meyer IH: Minority stress and mental health in gay men. J Health Soc Behav 1995;36:38-56.

19. Meyer IH: Prejudice, social stress, and mental health in lesbian, gay, and bisexual populations: Conceptual issues and research evidence. Psychol Bull 2003;129:674-697.

20. Mereish EH, Gamarel KE, Operario D: Understanding and addressing alcohol and substance use in sexual and gender minority youth. In: Brief Interventions for Adolescent Alcohol and Substance Abuse. Edited by Monti PM, Colby SM, O’Leary Tevyaw T. New York, NY: Guilford Press, 2018, pp. 305-327.

21. Watson RJ, Wheldon CW, Puhl RM: Evidence of diverse identities in a large national sample of sexual and gender minority adolescents. J Res Adolesc 2019 [Epub ahead of print]; DOI: 10.1111/jora.12488.

22. Puhl RM, Himmelstein M, Watson RJ: Weight-based victimization among sexual and gender minority adolescents: Findings from a diverse national sample. Pediatr Obes 2019; 14:e12514.

23. Robinson-Cimpian JP: Inaccurate estimation of disparities due to mischievous responders: Several suggestions to assess conclusions. Edu Res 2014;43:171-185.

24. Eisenberg ME, Gower AL, McMorris BJ, et al.: Risk and protective factors in the lives of transgender/gender nonconforming adolescents. J Adolesc Health 2017;61:521-526.

Address correspondence to: Ryan J. Watson, PhD

Department of Human Development and Family Sciences University of Connecticut 348 Mansfield Road, U-1058 Storrs, CT 06269

E-mail: ryanwatson@uconn.edu 